\title{
Cracking the Nut on LCME Standard 8.7: Innovations to Ensure Comparability Across Geographically Distributed Campuses
}

Johanna H. Foster, Julie Byerley, Heather Tarantino, Alice Chuang, Joseph Pino, Robyn Latessa, Suresh Nagappan, Rasheeda Monroe, Kurt Gilliland, Beat Steiner \& Gary L. Beck Dallaghan

To cite this article: Johanna H. Foster, Julie Byerley, Heather Tarantino, Alice Chuang, Joseph Pino, Robyn Latessa, Suresh Nagappan, Rasheeda Monroe, Kurt Gilliland, Beat Steiner \& Gary L. Beck Dallaghan (2019) Cracking the Nut on LCME Standard 8.7: Innovations to Ensure Comparability Across Geographically Distributed Campuses, Teaching and Learning in Medicine, 31:5, 544-551, DOI: 10.1080/10401334.2019.1609966

To link to this article: https://doi.org/10.1080/10401334.2019.1609966

+ View supplementary material 5
Published online: 18 Jun 2019.
Submit your article to this journal $\sqsubset$

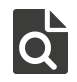

View related articles $\widetilde{\nearrow}$
View Crossmark data $\nearrow$ 


\title{
Cracking the Nut on LCME Standard 8.7: Innovations to Ensure Comparability Across Geographically Distributed Campuses
}

\author{
Johanna H. Foster ${ }^{a}$, Julie Byerley ${ }^{a}$, Heather Tarantino ${ }^{b}$, Alice Chuang ${ }^{a}$, Joseph Pino ${ }^{c}$ (D), Robyn Latessa ${ }^{d}$, \\ Suresh Nagappan ${ }^{e}$ (D), Rasheeda Monroe ${ }^{f}\left(\mathbb{D}\right.$, Kurt Gilliland ${ }^{a}$, Beat Steiner ${ }^{a}$, and Gary L. Beck Dallaghan ${ }^{a}$ \\ ${ }^{a}$ Office of Medical Education, University of North Carolina School of Medicine, Chapel Hill, North Carolina, USA; ${ }^{b}$ Department \\ of Medicine, University of North Carolina School of Medicine, Charlotte, North Carolina, USA; 'Departments of Internal Medicine \\ and Pediatrics, University of North Carolina School of Medicine, Wilmington, North Carolina, USA; ${ }^{\mathrm{d}}$ Department of Family

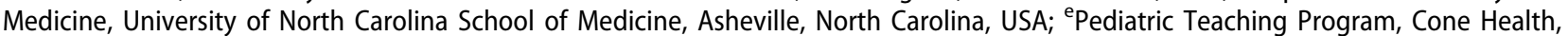 \\ Greensboro, North Carolina, USA; 'Department of Pediatrics, WakeMed Health and Hospitals, Raleigh, North Carolina, USA
}

\begin{abstract}
Problem: A large state university in the southeastern United States and state Area Health Education Centers (AHEC) collaborated to establish branch campuses to increase clinical capacity for medical student education. Prior to formally becoming branch campuses, two AHEC sites had established innovative curricular structures different than the central campus. These sites worked with the central campus as clinical training sites. Upon becoming formal campuses, their unique clinical experiences were maintained. A third campus established a curricular structure identical to the central campus. Little exists in the literature regarding strategies that ensure comparability yet allow campuses to remain unique and innovative. Intervention: We implemented a balanced matrix organizational structure, welldefined communication plan, and newly developed tool to track comparability. A balanced matrix organization model framed the campus relationships. Adopting this model led to identifying reporting structures, developing multidirectional communication strategies, and the Campus Comparability Tool. Context: The UNC School of Medicine central campus is in Chapel Hill. All 192 students complete basic science course work on central campus. For required clinical rotations, approximately 140 students are assigned to the central campus, which includes rotations in Raleigh or Greensboro. The remaining students are assigned to Asheville (25-30), Charlotte (25-30), or Wilmington (5-7). Chapel Hill and Wilmington follow identical rotation structures, 16 weeks each of (a) combined surgery and adult inpatient experiences; (b) combined obstetrics/gynecology, psychiatry, and inpatient pediatrics; and (c) longitudinal clinical experiences in adult and pediatric medicine. Asheville offers an 8month longitudinal integrated outpatient experience with discreet inpatient experiences in surgery and adult care. Charlotte offers a 6-month longitudinal integrated experiences and 6 months of block inpatient experiences. Aside from Charlotte and Raleigh, the other sites are urban but surrounded by rural counties. Chapel Hill is 221 miles from Asheville, 141 from Charlotte, and 156 from Wilmington. Outcome: Using the balanced matrix organization, various reporting structures and lines of communication ensured the educational objectives for students were clear on all campuses. The communication strategies facilitated developing consistent evaluation metrics across sites to compare educational experiences. Lessons Learned: The complexities of different healthcare systems becoming regional campuses require deliberate planning and understanding the culture of those sites. Recognizing how size and location of the organization affects communication, the central campus took the lead centralizing functions when appropriate. Adopting uniform educational technology has played an essential role in evaluating the comparability of core educational content on campuses delivering content in very distinct ways.
\end{abstract}

\section{KEYWORDS}

accreditation; comparability; longitudinal integrated clerkships;

regional campuses

\section{Background}

Regional medical campuses have been well described in the literature and are increasing in number. ${ }^{1-3}$
Branch campuses offer certain benefits, such as broad service to the state, expansion of clinical teaching opportunities, curriculum innovation, efficient medical

CONTACT Gary L. Beck Dallaghan, Ph.D. @ gary_beck_dallaghan@med.unc.edu O Office of Medical Education, 311 Berryhill Hall, CB\#7321, Chapel Hill, NC, 27599-7321, 919962 3403. @ @GLBDallaghan; @UNC_SOM 
education cost structure, and distributed physician workforce development, especially to less populated areas. $^{4-6}$ The University of North Carolina is a large public medical school with three established regional campuses for clinical education and the potential to grow at least two more. All students complete the 15month preclinical courses (Foundation Phase) in Chapel Hill and then are assigned to a campus for the 1-year core clinical experiences (Application Phase). Students assigned to regional campuses have the option to remain at their campuses for the 14-month final phase (Individualization Phase) or move between the campuses to complete graduation requirements.

Established in 1879 initially as a 2-year program, the University of North Carolina School of Medicine (UNC SOM) serves the citizens of North Carolina by preparing the next generation of physicians. ${ }^{7}$ The medical school is supported by numerous community clinical teaching sites across the state working through the North Carolina Area Health Education Centers (NC AHEC). The NC AHEC was established in 1971 through federal and state funding to support community-based rotations for medical students and to deliver clinical services to rural and underserved areas. Although there are nine AHEC used for medical education and patient care in North Carolina, the UNC SOM heavily relies on five of them to allow students to experience clinical care from the state's western mountains to its eastern coast.

The UNC SOM formalized relationships with two NC AHEC sites by establishing regional campuses located in Asheville and Charlotte in 2009 and 2010, respectively. The campuses were established to take advantage of the high-quality teaching in each site and to more effectively develop a pipeline of physicians for those areas. Both campuses, with their longitudinal integrated clerkships, quickly became popular with students based on student evaluations. Students with rural interests could pursue their clinical training in the Asheville campus's mountain setting, and students with urban interests can choose the Charlotte campus's cosmopolitan setting of 2 million people. In March 2016, the UNC SOM opened its third campus in Wilmington, a city of 100,000 in southeastern North Carolina that supports a rural coastal population culturally different from the mountain population of Asheville. In addition to spending time at UNC Hospitals and local clinics, students based on the school's Central Campus in Chapel Hill also complete clinical rotations in nearby Greensboro and Raleigh.

North Carolina is home to both well-known thriving urban centers like Charlotte and Raleigh and vast rural areas. Eighty of North Carolina's 100 counties are classified as rural according to metrics on population density, median income, employment rates, and health outcomes. Each of UNC SOM's campuses reflect this urban and rural divide, allowing students to experience medical education in unique environments.

Asheville is located in urban Buncombe County, which is surrounded by counties classified as rural due to low population densities of fewer than 250 people per square mile. This area of the state lacks access to healthcare with approximately four to nine primary care physicians per 10,000 population and experiences some of the worst health outcomes in the state (see the NC Rural Center; https://www.ncruralcenter.org/). ${ }^{8}$ As such, the healthcare system in Asheville serves as a major access point for healthcare in the western part of the state.

Asheville pioneered the longitudinal education model for the UNC SOM by adopting the Harvard Cambridge model. At this campus, students have 8 months of integrated outpatient experiences; they track the same patients, progressively assuming more responsibility in their care. The balance of the year are discreet inpatient experiences that mirror traditional clerkships in surgery and inpatient adult care. Notably, this campus was the first to adopt a humanities curriculum that has since been adopted throughout the school.

In contrast, Charlotte is located in urban Mecklenburg County, a classification that extends to its neighboring counties due to their connections to the finance and banking industry. Although this area of the state has greater access to primary care and specialty physicians, it is also home to large populations of African American and Hispanic residents who experience higher rates of health disparities compared to White residents. ${ }^{9}$ Medical students in Charlotte have the opportunity to serve urban underserved patients who may not be native English speakers. Charlotte adopted an integrated longitudinal model but modified the duration of it. Students experience a 6-month integrated outpatient longitudinal experience and then complete 6 months of inpatient services that mirror traditional clerkships.

The Wilmington Campus is located in urban New Hanover County neighbored by rural counties, with a healthcare workforce supply and health outcomes that mirror those of the western part of the state. Unique to the eastern part of the state is its notable military presence, with large marine corps, army, and air force bases in close proximity to the Wilmington Campus, 
allowing students to interact with active military members and veterans. Wilmington Campus's curriculum is identical to that of the central campus, which is organized into 16-week trimesters of integrated courses. There are three clinical courses and one longitudinal humanities course. Hospital, Interventional, and Surgical Care delivered a combined surgery and adult inpatient care course. Care of Special Populations is a combination of three core clerkships. This course rotates students in both inpatient and outpatient settings for obstetrics/gynecology and psychiatry while solely focusing on inpatient pediatric care. Community-Based Longitudinal Care provides students training experiences in outpatient adult and pediatric care with specialty opportunities, such as Hospice.

Whereas regional campuses benefit communities, schools, and learners, ${ }^{10}$ medical schools must make certain that students receive comparable educational experiences at each campus. This becomes even more challenging when you have regional campuses that could be described as a clinical model or longitudinal/ distributed model. ${ }^{2}$ Comparability involves setting and communicating expectations for clinical education, engaging stakeholders for input, and systematic data collection and reporting with benchmarks. ${ }^{11}$ The Liaison Committee on Medical Education (LCME) has specific standards for medical schools with one or more geographically distributed campuses, ${ }^{12}$ specifically defined as comparable educational experiences, equivalent methods of assessment across all locations, equivalent student resources, and a uniform graduation standard. This particular element of the LCME Standards has been cited as one of the more common variables resulting in severe action by the LCME. ${ }^{13}$ For the almost 50 medical schools that now have geographically distributed campuses these standards can be challenging because of distance, diffuse faculty relations, and regional campuses' need for autonomy. ${ }^{14}$ Little has been written about how to overcome these obstacles.

We describe a process to overcome the challenges of maintaining the integrity and comparability of the clinical curriculum through the three campuses in Asheville, Charlotte, and Wilmington in addition to the Central Campus in Chapel Hill, which includes rotations in Greensboro and Raleigh. The challenge comes not only from the unique locations but also from the curricular structure at each site. Evaluating comparable experiences while respecting the unique features of each campus required developing a framework that allowed us to quickly compare sites. ${ }^{15}$

\section{A balanced matrix organization}

The central campus approached issues of comparability as a challenge of managing a balanced matrix organization with its various components that have dual reporting responsibilities to local host institutions (sponsoring hospitals and local AHEC) and to the School of Medicine with its obligations to the LCME. ${ }^{12}$ The definition and principles of a Balanced Matrix Organization come from the business literature, in which a manager has two or more upward reporting lines in order to manage large and complex projects. ${ }^{16,17}$ This model is common in the business world because it recognizes that expertise does not reside in any one unit of an organization, promotes efficiency due to combined resources, and facilitates shared leadership toward common goals.

For the UNC SOM, its geographically distributed campuses and partnerships with AHECs represent a level of complexity that required the Balanced Matrix Organization. This model established a framework by which lines of communication and decision-making could be made collectively and locally. Functionally, applying this model established inter- and intracampus communication structures for curricular issues. This framework also helped conceptualize the interand intracampus lines of communication with medical school leadership and health system leadership.

At the same time, there are potential challenges associated with the Balanced Matrix Organization, including the confusion created by two bosses, muddled flow of information, and competing priorities. The school took these challenges into account when applying the Balanced Matrix Organization by establishing clear reporting structures, communication channels, and accountability measures. Next we describe how we optimized this model in our work developing the regional campuses.

\section{Clear reporting structure}

Clarity in the organizational structure is essential. Each campus has an assistant dean who reports to the central SOM Executive Vice Dean for Education or the Senior Associate Dean and oversees the educational roles of local faculty. Site directors are established for each course at each branch campus, and those site directors report to the centrally based Chapel Hill course director for curriculum-specific 


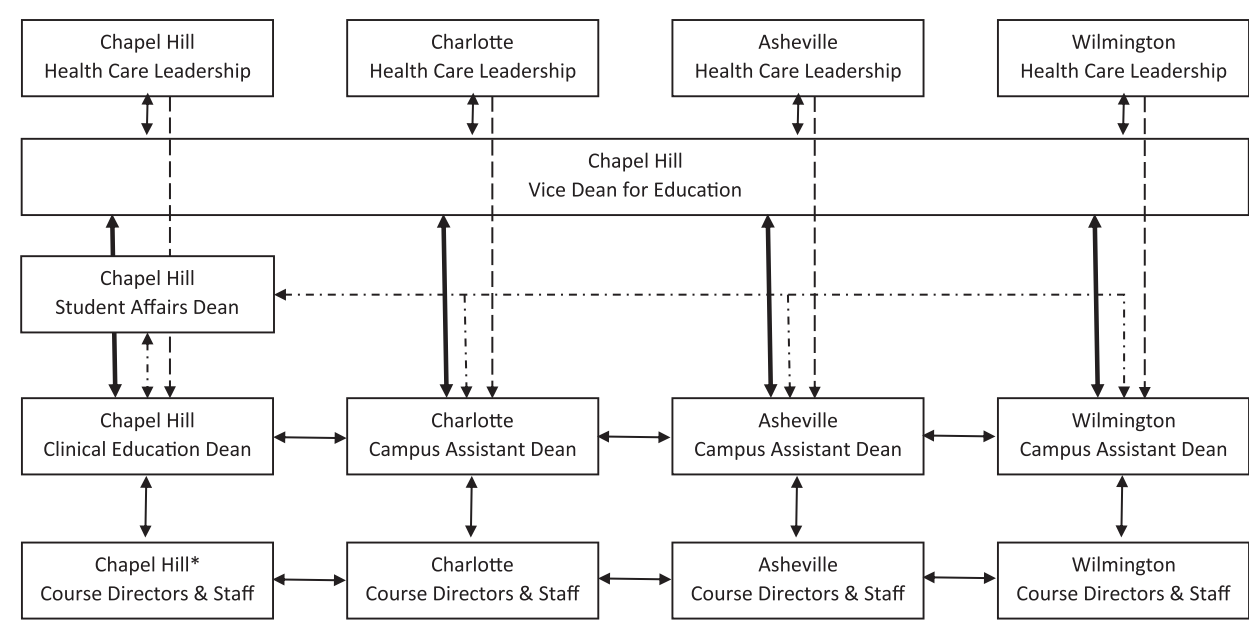

Figure 1. Matrix Organization Connecting the Central Campus with the Regional Campuses. Note: There are site directors located on each campus; however, the Chapel Hill course director is designated as the primary course director who is responsible for all final grades.

information, such as learning objectives, assessments, and final grades (Figure 1). The site directors also report to the Campus Assistant Dean on day-today issues.

Per LCME Standards, ${ }^{12}$ all faculty need appointments at the UNC SOM, but, even more than formal appointments, faculty from regional campuses need to be involved in the educational activities of the central campus. In our setting, this is accomplished by Campus Assistant Dean participation on committees that are essential to the education functions of the school. All Campus Assistant Deans sit on our Dean's Advisory Committee, Education Committee, and the Application and Individualization Phase curriculum committees. They may also serve on the admissions, mistreatment, and relevant ad hoc committees. A single graduation standard is ensured through the use of a single promotions committee who evaluates all students using a unified set of six core competencies.

It is important to avoid the perception of a tiered system among the campuses. For that reason, we have actively chosen to admit all students centrally for the Foundation Phase, then allow students to be matched to their clinical campus midway through their 1st year. Students are encouraged to do campus visits during the 1st year and then are allowed to rank their choices. The goal is to optimize campus capacity while prioritizing student preference. Students convey their preferences by ranking each campus and submitting a short essay, which the school takes into account when making assignments. Using this method for the past 3 years, we have been able to award $92 \%$ or more of students their first choice, and the remainder are awarded their second choice due to over- or undersubscription of a campus. We find this assignment strategy the optimal blend of creating a single unified class and accommodating student request to the greatest extent possible.

\section{Well-defined communication plan}

The most significant challenge faced in the regional campus model is the balance of autonomy for each campus with consistency throughout the school. Each setting has its own culture and pride, and we have worked to ensure that each regional campus feels respected and valued. Because two of our campuses were well-established clinical training sites before formally becoming regional campuses, consideration of how we communicated as a school of medicine needed to honor those legacies while presenting a unified school of medicine. This requires ongoing attention and committed communication, as well as local commitment to the educational mission.

Overcoming the geographic distances between the regional campuses has been a major challenge. It takes 7 hours to drive from the campus in the east to the campus in the west. Without a well-defined multidirectional communication plan, leaders, educators, and staff at the regional campus were often not informed of decisions in a timely manner and did not have sufficient opportunities for input on major issues. A combination of planned face-to-face meetings and videoconferencing that includes members at all levels of the organization has helped.

Videoconferencing is now expected at each of our formal Education Committee meetings. Campus assistant deans are able to participate in all clinical curriculum committees in this manner. Campus 
assistant deans, key educators, and staff also participate in monthly conference calls with the education deans. In the past, each campus used different telecommunications technologies, generating technical difficulties that impeded communication and elevated frustration.

To remove these barriers, the school invested in uniform, secure telecommunications software and hardware that the central department of information technology vetted and installed. Members of this department also trained campus representatives to ensure optimal use. After installation and training, we improved and simplified the audio and video infrastructure in multiple conference rooms across the campuses, allowing for planned and impromptu communication.

Although video conferencing is important, we have found that face-to-face meetings remain vital for strategic discussions or complex problem solving. Core educators and staff members from all campuses meet formally four times a year. Location of the meetings rotates to one of the four campuses and two major clinical sites, Raleigh and Greensboro. These campus leadership meetings are 2-day events that include a celebratory social aspect. In addition, education deans and staff members visit each branch campus regularly, sometimes hosting meetings video conferenced from that site.

Frequent communications and deliberate attention to maintaining a balanced matrix organization have helped build trust that is allowing us to tackle some difficult issues. For instance, although not a standard of the LCME, establishing a culture of trust and open communication is essential for success with distributed campuses. With that in mind, it is critical that the central campus take pride in its regional sites, recognizing their important contributions to its mission, and that each campus takes ownership of its role in the success of the school as a whole. We felt it important to refer to the central campus as "Chapel Hill" rather than "UNC" because each campus is UNC SOM. The use of inclusive language such as "our students/faculty on the Asheville campus" rather than "your students/faculty in Asheville" when referring to those at a regional sites reinforces that we are all one school. Likewise, we have developed consistent campus logos and web pages for identification. Students also have the opportunity to participate in class meetings using telecommunications. Student government has specifically addressed this as well, with a "Bonding Across the Miles" initiative that ensures that major SOM social events (such as skit night and the student gala) are planned to optimize student participation regardless of campus. It is important that there is a clear vision of one large school with one distributed student body.

\section{Accountability through comparability measures}

We established our reporting structure and communication plan in applying the Balanced Matrix Organization to manage the complexity of a multiregional educational mission. However, to fully optimize the Balanced Matrix Organization, we needed accountability measures to maintain focus in environments with competing priorities. ${ }^{11}$ Each year, the Education Committee, the school's top governing structure, establishes improvement plans. As a step toward a better Balanced Matrix Organization, the 2015-2016, Improvement Plan prioritized achieving campus comparability for the clinical Application Phase by establishing uniform standards, metrics, and services.

An instrument we call the Campus Comparability Tool (Supplementary Figure 1) was developed by our Education Committee for tracking measures of comparison. The measures included in the tool were derived from the LCME standards addressing comparability of regional campuses. ${ }^{12}$ Given the unique attributes of each campus, referring to these standards allowed us to reach consensus while acknowledging contextual factors at each site. ${ }^{15}$ In addition, objective academic outcomes along with subjective feedback from students' measures can be incorporated into the instrument to add dimension to data.

Next we present how the tool provided data categories used to demonstrate comparability, how to operationalize data collection, and examples of data collected. We also describe examples of increased comparability and collaboration that did not derive directly from the use of the tool but stemmed from increased trust built by frequent and open communication.

\section{Common educational core and clinical experiences}

A fundamental expectation of comparability is that students across geographic campuses meet a single graduation standard and achieve the same set of core competencies specified by the institution, in our case through the Education Committee. The Foundation Phase curriculum is delivered at the central campus for all students. The regional campuses have parallel yet individualized clerkship 
curricula for the Application Phase that allow use of the strengths of the communities in which they are based. ${ }^{18}$ At the same time, branch campuses often serve as incubators, developing and testing innovative educational practices that are later generalized to the entire school. Granting branch campuses flexibility has enabled the school to broaden its pool of creativity and empowered the campus to play a role in shaping the overall curriculum. One example is the ethics and humanism course originating in Asheville with content being adopted by the Chapel Hill campus.

For each curriculum, the same medical education core program objectives, the same required minimum number and type of clinical encounters, and the same assessment measures were developed collaboratively with representation from each campus. Campuses and parallel curricula may vary in their method of delivery to teach objectives. They may also have additional objectives and assessments beyond the core to allow differentiation and take advantage of the unique environments and strengths of each campus. Maintaining a focus on a core educational mission while allowing flexibility for campuses to implement different curricular models represents a way to support innovation. Supplementary Figure 1, Items 1 and 2, provide summary data for the campuses, highlighting subtle differences between them.

\section{Common assessment tools and grading schema}

All site directors work together to agree upon course objectives that address the UNC SOM Core Competencies and to build assessments that will ensure these are met. Assessments may be administered and/or graded centrally or at NC AHEC sites depending on resources available. If they are administered locally, site directors work closely to develop processes to ensure the assessment achieves identical goals, as well as institute grade data and standard setting processes are put in place to ensure fair evaluation (Supplementary Figure 1, Items 3 and 4).

Students return to the central campus for assessment using objective structured clinical exams. Chapel Hill-based Course Directors provide central oversight over many curricular aspects, including grade assignments. They conduct end-of-course conference calls with site directors to finalize grades according to a mutually agreed-upon grade distribution.

All campuses use the same evaluation software to collect grades and manage a multitude of evaluations, including internally developed student surveys. The use of a common evaluation software and dedicated staff have allowed the school to centralize program evaluation efforts that are critical to measuring and monitoring comparability. Central Campus has also taken the lead in establishing school-wide policies that take campus procedures into account, as well as developing response protocols for reports of mistreatment.

However, not every function requires centralization; doing so would inhibit the development of unique campus characteristics and its ability to test new educational practices. For instance, the Asheville campus pioneered the development and implementation of a social and health systems curriculum that focused on ethics, public health, and humanities. This aspect of the Asheville curriculum became a defining aspect of its program along with its 8-month longitudinal clinical curriculum. Since Asheville's rollout of the social and health systems curriculum, the school has established a required course for all core clinical students at every campus. The Charlotte campus has also contributed to school-wide curricular change when it implemented its ultrasound curriculum, a characteristic that attracted many students to its program. Ultrasound has fast become an essential diagnostic tool, and the Charlotte campus understood its importance and trajectory in 2013. It created a forward-thinking curriculum to equip students for the broad use of this tool, which the school is working to build upon by creating an ultrasound curriculum for all campuses. Finally, the Wilmington campus launched a physician leadership program in 2016 with the aim of providing students with key management skills. This campus worked with its local university's business school and AHEC to create a curriculum, which has since spurred a school-wide effort to formalize a leadership curriculum for all students beginning in their first semester of medical school. Given their smaller cohorts and scopes of operation, they are more nimble at developing, implementing, and adjusting ideas. The school continually works to balance the application of centralization, blended responsibility, and local control.

Although the timing of student feedback evaluations varies according to curricula at UNC SOM, their organization by medical discipline does not. The school learned this lesson after the first year of the Application Phase, when efforts to aggregate data were delayed because the Asheville and Charlotte campuses collected student feedback on a single evaluation that combined inpatient and outpatient experiences reflective of their longitudinal structure. In contrast, Central Campus and Wilmington students provide distinct feedback for inpatient and outpatient experiences. For the following academic year, Central 
Campus staff worked with branch campus staff to separate the evaluation of experiences according to clinical settings. In short, incoming data affect outgoing aggregated data, and schools with branch campuses must be mindful of these curricular differences when constructing evaluation instruments and comparability metrics.

\section{Commitment to learners}

A positive learning environment is vital for student success. Because the learning environment impacts students across all 4 years, a centralized mechanism was established for learners to report concerns. Campus leadership is committed to addressing issues that arise as well as discussing issues on regular teleconference meetings with the Associate Dean for Student Affairs (Supplementary Figure 1, Item 5).

Academic support services are available on our central campus and at AHEC campuses. All students are assigned an undifferentiated advisor on the Central Campus at the start of medical school. This advisor actively works with each student, regardless of campus, each semester throughout their time at UNC. When students go to their regional campus, they are assigned advisors at that site. Career advising is available locally at the campuses but is accomplished with the help of faculty development from the central campus, shepherded by our Associate Dean for Student Affairs' office (Supplementary Figure 1, Item 6). For specialties that are not well represented at local campuses, additional career advising support is available from the Central Campus. Students are excused from clinical and academic duties to meet with advisors when necessary.

The LCME Standards also expect that medical schools provide comparable academic support, career advising, educational records, medical student health services, personal counseling, and financial aid services. ${ }^{19}$ Some student services, such as financial aid and educational records management, can be operationalized centrally. Student health services, including counseling resources, must be available locally (Supplementary Figure 1, Item 7). At UNC SOM, we contracted with specific organizations to ensure that our students could use local services in each regional campus area, taking advantage of local state university resources if available. For example, students on the Wilmington campus of the SOM are allowed to use student health resources at the University of North Carolina Wilmington.

\section{Discussion}

Balancing the need for campus comparability with practical implementation is an ongoing challenge. Geographic distance, institutional cultures, and varying curricular structures have the potential to present barriers for comparable educational experiences. Multicampus medical schools require the flagship campus to take a lead in centrally developing and managing evaluation, key functions, and policies while continually facilitating inclusive decision-making.

The adoption of the Balanced Matrix Organization model allowed us to harness the opportunities of a multicampus school of medicine by applying structure. This model specifically allowed the school to reach decisions on common educational goals, methods, student assessment, and outcomes while weighing the campus educational strengths, resources, passions, and costs. Stakeholders at each campus are integral to decision-making due to the communications plan that was implemented, which has strengthened relationships to allow for ongoing regular dialog within meetings and for just-in-time needs. Although data were being centrally collected on student performance from each site, the data were not summarized in a systematic and organized way to allow easy comparison across campuses. The development of the Campus Comparability Tool provided structure that can be readily adapted by other institutions to compare sites.

With these comparisons available, the campus educational leaders are able to detect and discuss discrepancies from one site to the other. Being accountable for the educational experiences is a component of comparability raised by Chandran. ${ }^{11}$ Without the ability to easily compare data across sites, the flagship campus may be unaware of sites that may be underperforming. Our Campus Comparability Tool provides important metrics to focus continuous quality improvement at each site.

\section{Conclusion}

The branch campus system is an efficient and effective way for medical schools to serve their states, increase clinical capacity for medical students, pilot innovation, and enhance the workforce across multiple areas of need. ${ }^{10}$ Achieving comparability, although challenging, is essential to accreditation and can be accomplished through structural, social, and evaluative efforts. ${ }^{15}$ The matrix organization we use can be implemented at other schools to ensure active engagement of all the campuses. ${ }^{16}$ The deliberate communication 
strategy, which ensures frequent interactions between the campuses and helps avoid misunderstandings, can be used by other schools. The Campus Comparability Tool included here was developed to track this process at UNC SOM and can be adapted to other institutions seeking multiple measures of consistency across sites.

\section{Acknowledgments}

We thank Don Pathman, MD, for his thoughtful review of the manuscript and the additional members of the UNC SOM Education Committee who participated in the development of the Campus Comparability Tool.

\section{Funding/support}

None

\section{Conflict of interest}

The authors declare they have no conflicts of interest for this study.

\section{Ethical approval}

Not applicable

\section{ORCID}

Joseph Pino (D) http://orcid.org/0000-0002-3656-0005

Suresh Nagappan (D) http://orcid.org/0000-0003-0290-7450

Rasheeda Monroe (iD http://orcid.org/0000-0002-0454-8837

Gary L. Beck Dallaghan (D) http://orcid.org/0000-00028539-6969

\section{References}

1. Mallon WT, Lui M, Jones RF, Whitcomb M. MiniMed: The Role of Regional Campuses in U.S. Medical Education. Washington, DC: Association of American Medical Colleges; 2003.

2. Cheifetz CE, McOwen KS, Gagne P, Wong JL. Regional medical campuses: a new classification system. Acad Med. 2014;89(8):1140-1143. doi:10.1097/ ACM.0000000000000295.

3. AAMC Group of Regional Medical Campuses. https:// www.aamc.org/members/grmc/resources/. Last accessed January 6, 2018.

4. Norris TE, Coombs JB, House P, Moore S, Wenrich MD, Ramsey PG. Regional solutions to the physician workforce shortage: the WWAMI experience. Acad Med. 2006; 81(10):857-862. doi:10.1097/01.ACM.0000238105.96684.2f.

5. Mallon WT, Lui M, Jones RF, Whitcomb M. Regional Medical Campuses: Bridging Communities, Enhancing Mission, Expanding Medical Education. Washington, DC: Association of American Medical Colleges; 2006.
6. Cathcart-Rake W, Robinson M, Paolo A. From infancy to adolescence: the Kansas university school of medicinesalina: a rural medical campus story. Acad Med. 2017; 92(5):622-627. doi:10.1097/ACM.0000000000001455.

7. Bryan J, McLendon W, Savage K. Medicine at Chapel Hill: The Department of Medicine at the University of North Carolina 1952-2007 Chapel Hill, NC: BW \& A Books; 2012.

8. North Carolina Health Professions Data System. Program on Health Workforce Research and Policy, Cecil G. Sheps Center for Health Services Research, University of North Carolina at Chapel Hill. Available at https://nchealthworkforce.unc.edu/supply/. Accessed May 12, 2019.

9. MCHD Epidemiology Program. Health Disparities \& Inequities: Mecklenburg Residents. Available at https:// www.mecknc.gov/HealthDepartment/HealthStatistics/ Documents/Health\%20Disparities\%20and\%20Inequities \%2c\%20Mecklenburg\%20County\%20Residents.pdf. Accessed May 12, 2019.

10. Snadden D, Bates J, Burns P, et al. Developing a medical school: expansion of medical student capacity in new locations: AMEE Guide No. 55. Med Teach. 2011; 33(7):518-529. doi:10.3109/0142159X.2011.564681.

11. Chandran L. Ensuring comparability of educational experiences during clinical clerkships at multiple sites. Curriculum Inventory in Context 2015;2(5). https:// www.aamc.org/download/464768/data/ciic02-5May2015. pdf. Accessed February 9, 2019.

12. LCME standards. http://lcme.org/publications/. Last accessed January 6, 2018.

13. Hunt D, Migdal M, Waechter DM, Barzansky B, Sabalis RF. The variables that lead to severe action decisions by the Liaison Committee on Medical Education. Acad Med. 2016;19:87-93. doi:10.1097/ ACM.0000000000000874.

14. The Evolving Landscape of Regional Campus Development- Discussion Notes. AAMC GRMC Meeting; 2014. https://www.aamc.org/download/ 423452/data/grmcbusinessmeetingtablediscussionnotes. pdf. Last accessed January 6, 2018.

15. Hautz SC, Hautz WE, Feufel MA, Spies CD. Comparability of outcome frameworks in medical education: implications for framework development. Med Teach. 2015;37(11):1051-1059. doi:10.3109/ 0142159X.2015.1012490.

16. Galbraith JR. Designing Matrix Organizations That Actually Work. San Francisco, CA: Josey-Bass; 2009.

17. Stuckenbruck LC. The matrix organization. Project Management Quarterly. 1979;10(3):21-33.

18. Hansen L, Simanton E. Comparison of third-year student performance in a twelve-month longitudinal ambulatory program with performance in traditional clerkship curriculum. S D Med. 2009; 62(8):315-317.

19. Kollhoff L, Kollhoff M, Cathcart-Rake W. Providing support services for medical students on a rural regional medical campus. Medscieduc. 2015;25(2): 157-162. doi:10.1007/s40670-015-0108-8. 